\title{
A UNIFORM MODEL OF THE MASSIVE SPINNING PARTICLE IN ANY DIMENSION.
}

\author{
S. L. Lyakhovich, A. A. Sharapov and K. M. Shekhter \\ Physics Department, Tomsk State University, 634050 Tomsk, Russia
}

\begin{abstract}
The general model of an arbitrary spin massive particle in any dimensional spacetime is derived on the basis of Kirillov - Kostant - Souriau approach. It is shown that the model allows consistent coupling to an arbitrary background of electromagnetic and gravitational fields.
\end{abstract}

PACS codes: 02.20.-a; 11.30.Cp; 03.65.pm; 11.90.+t.

Keywords: spinning particles, Poincaré group, orbit method, constrained dynamics, geometric quantization.

\section{Introduction}

The Lagrangian description of the relativistic spinning particles is one of the recurrently discussed themes in high energy physics, having a long history. The retrospective exposition of the question and some basic references can be found in the review [罒. In the context of $M$-theory the models of spinning particles awake today fresh interest as special but highly nontrivial examples of 0-branes which along with some other extended objects are considered to be the basic ingredients of the non-perturbative string theory [2]. Since the target space of a consistent string theory has certainly more than four dimensions (10 or perhaps 11) the observable space-time is supposed to result from the Kaluza-Klein compactification of extra dimensions in the low energy limit. In so doing the effective 
dimension of p-brane may decrease down to zero when it is considered from the viewpoint of four-dimensional observer. The classical example is the double dimensional reduction of $d=11$ supermembrane to the type II A string in $d=10$ [3]. This opens up an interesting possibility to interpret the spinning particles as low energy effective models of the p-branes in higher-dimensional space-time, which compact directions are associated with the spinning degrees of freedom of the particle.

All this gives rise to the question about the construction of the mechanical models of relativistic spinning particles in the space-time of arbitrary dimension. The reach kinematical symmetries underlying the models enable one to treat them as elementary dynamical systems in the Souriau sense [4] and to apply for their description the full machinery of the symplectic geometry. (For applications of this approach see also [5]). In the framework of this scheme the whole dynamical information about the space-time and phase-space evolution of the system is encoded in a presymplectic manifold $\mathcal{E}$ being a homogeneous transformation space of the group $G$ for which the system is elementary one. For a given physical system, the choice of $\mathcal{E}$ is very ambiguous and there is no precise prescription for it. Fortunately, there is no matter how to choose particular $\mathcal{E}$ when describing free particle: any $\mathcal{E}$ leads to the proper classical dynamics

Various models of spinning particles are known with $4 d$ symmetry groups: Poincaré [6, 7], de Sitter [8] and Galilei [9]. The $3 d$ and $6 d$ analogues to these models can be found in ref. [10, 11], for superextensions see [12, 13]. The covariant operatorial quantization of these models leads to the Hilbert space of physical states carrying the unitary irreducible representation of the respective groups. Some of these models have, however, a common problem of constructing a consistent extension to the case of spinning particle subject to exterior fields. The obstacle is that the space-time dynamics of the particle, arising in these models in some cases, is characterized by two-dimensional world-tubes rather then world-lines. The non-local behavior of such a type, sometimes referred to as the phenomenon of Zitterbewegung [14], is usual for the relativistic particles with spin and presents the main obstruction to the switching on a local interaction. Note that the difficulty is not inherent to these systems, as they are, but it is rather related to the way

${ }^{a}$ For example, one can always identify $\mathcal{E}$ with the underlying symmetry group $G$ itself. 
of their description, which basically depends on the choice of $\mathcal{E}$. In particular, the model of the ref [7], in $d=4$, does not display the Zitterbewegung, and it allows the consistent interaction.

In recent paper [15] we have constructed the model for a massive particle of integer spin living in $d$-dimensional space-time and coupled to an arbitrary background of gravity and electromagnetism. The underlying presymplectic manifold was identified with that for the spinless particle times a regular (co)adjoint orbit of space rotations group

$$
\begin{gathered}
\mathcal{E}=\mathcal{E}_{\text {spinless }} \times \mathcal{O}_{\mathbf{s}} \\
\mathcal{E}_{\text {spinless }}=\mathbf{R}^{d-1,1} \times B, \quad \mathcal{O}_{\mathbf{s}}=S O(d-1) /[S O(2)]^{r}
\end{gathered}
$$

Here $r=\operatorname{rank} S O(d-1)=[(d-1) / 2]$ and $B$ stands for the upper sheet of the mass hyperboloid: $p_{A} p^{A}=-m^{2}, \quad p_{0}>0$. The regularity of $\mathcal{O}_{\mathbf{s}}$ means that the whole space of invariant presymplectic structures on $\mathcal{E}$ is parametrized by $r+1$ numbers $m, \mathbf{s}=\left(s_{1}, s_{2}, \ldots s_{r}\right)$ associated with mass and spin(s) of the particle . The space-time motion of the particle is described here by the one-dimensional world-lines (time-like geodesics of Minkowski space) and, as a result, the model is free from the above mentioned obstacle to the interaction. For the sake of explicit Poincaré covariance, the orbit $\mathcal{O}_{\mathrm{s}}$ was symplectically embedded into $\oplus_{i=1}^{r} \mathbf{R}_{\mathbf{C}}^{d-1,1}$ equipped with the natural action of the Lorentz group $S O(d-1,1)$ and invariant symplectic form. This realization for $\mathcal{O}_{\text {s }}$ proves to be especially suitable for the covariant quantization of integer-spin particle but it becomes inadequate when trying to consider half-integer spins since the quantum-mechanical description for the latter case is based on the group $\operatorname{Spin}(d-1,1)$ rather than $S O(d-1,1)$. In order to take into account the half-integer spins, one should replace, from the very beginning, the proper Lorentz group $S O(d-1,1)$ by its double covering $\operatorname{Spin}(d-1,1)$.

In this paper we propose the new construction for the spinning sector of the massive

\footnotetext{
${ }^{b}$ This is just the number of parameters labelling a general massive representation of Poincare' group. Indeed, fixing mass of the particle reduces the classification problem for the Poincare' group representations to the one for the Wigner little group $S O(D-1)$. According to the general Borel-Bott-Weil theory [16], there is a one-to-one correspondence between regular (co-)adjoint orbits of the orthogonal groups and theirs representations. The use of the other (irregular) orbits would lead only to the special spin representations, which can be obtained in our model by specifying values of $\mathbf{s}^{\prime}$.
} 
particle which provides a uniform quantum-mechanical description for both integer and half-integer spins. For these ends, the phase space of spinning degrees of freedom $\mathcal{O}_{\mathrm{s}}$ is

embedded into the carrier space of the Lorentz group $\mathbf{C}^{2^{[d / 2]}} \oplus_{i=1}^{r-1} \mathbf{R}_{\mathbf{C}}^{d-1,1}$, where the first factor transforms under the spinor representation. Then the (half-)integer spin representations may be obtained by applying either geometric or Dirac quantization to this model. A remarkable property of this realization for $\mathcal{O}_{\mathbf{s}}$ is that the use of spinor variables makes possible to resolve the mass-shell condition $p_{A} p^{A}=-m^{2}$ in a Lorentz-invariant manner. It can be thought about as an extension to the massive case of the twistor realization known for the isotropic momenta of a massless particle in special dimensions: 3, 4, 6 and 10. The distinction is that the spinor variable carries now an information about both the mass hyperboloid $B$ and the internal space for spin $\mathcal{O}_{\mathbf{s}}$. The details of this construction are presented in the next section together with the generalization to the case of minimal coupling to exterior gravitational and electromagnetic fields.

In Section 3, we reformulate the model as Hamiltonian system with the first and second class constraints and study the physical spectrum of the theory within the Dirac quantization scheme. The physical wave functions, being extracted by the quantum operators assigned to the constraints, are shown to be in one-to-one correspondence with the Poincaré-irreducible (spin-)tensor fields in Minkowski space.

We conclude the paper by discussing the obtained results and some further perspectives.

\section{Classical description}

As it was mentioned in the previous section the extended phase space of the massive spinning particle is constructed to be the direct product of the extended phase space of the spinless particle and the manifold $\mathcal{O}_{\mathbf{s}}$, responsible for spinning degrees of freedom. The former factor is standardly embedded into the cotangent bundle $T^{*}\left(\mathbf{R}^{d-1,1}\right)$ of the Minkowski space by the mass-shell condition

$$
p_{A} p^{A}+m^{2}=0
$$


while the latter is identified with so-called flag manifold, which points may be viewed as the sequences

$$
\begin{aligned}
& 0 \subset V_{1} \subset V_{2} \subset \ldots \subset V_{r} \subset \mathbf{R}_{\mathbf{C}}^{d-1,1}, \\
& p \perp V_{k}, \quad \operatorname{dim} V_{k}=k
\end{aligned}
$$

of complex $p$-transversal vector subspaces of complexified Minkowski space, telescopically embedded into each other. In the previous paper [15], we have suggested the holomorphic parametrization for $\mathcal{O}_{\mathrm{s}}$ with the help of $r$ independent complex vectors $Z_{i}^{A} \subset \mathbf{R}_{\mathbf{C}}^{d-1,1}, i=$ $1,2, \ldots r ; A=0,1, \ldots d-1$ subject to the conditions

$$
\left(Z_{i}, Z_{j}\right)=0, \quad\left(p, Z_{i}\right)=0, \quad Z_{i}^{A} \sim Z_{j}^{A} \Lambda_{i}^{j},
$$

where $\Lambda_{i}^{j}$ is a complex non-degenerate upper-triangular $r \times r$ matrix, and $(\ldots, \ldots)$ denotes the inner product with respect to the Minkowski metric $\eta_{A B}=\operatorname{diag}(-,+\ldots,+)$. In this realization, each $V_{k}$ is spanned by the vectors $Z_{1}^{A}, Z_{2}^{A}, \ldots Z_{k}^{A}$ and the last relation in (3) establishes the equivalence between all such frames in $V_{k}$.

To construct a spinor realization for $\mathcal{O}_{\mathbf{s}}$, it is sufficient to parametrize a subspace $V_{r}$ by a spinor variable. Consider the commuting Dirac spinor $\psi_{a}, a=1, \ldots 2^{[d / 2]}$ subject to the following constraints and equivalence relations?

$$
\begin{gathered}
\psi_{a} \sim \lambda \psi_{a}, \quad \lambda \in \mathbf{C} \backslash\{0\} \\
p_{A} \Gamma_{a}^{A b} \psi_{b}=m \psi_{a}, \quad Z_{k A} \Gamma_{a}^{A b} \psi_{b}=0, \quad k=1, \ldots r
\end{gathered}
$$

Since some subsequent expressions may differ for the cases of even- and odd dimensions, the formulae will be labeled with the letters $a$ and $b$ for the former and latter case respectively. Accounting (4) and the Fierz identities, the spinor bilinear is decomposed in the basis of the Clifford algebra generated by $\Gamma$-matrices as follows:

$$
\psi \otimes \widetilde{\psi}=M_{A(r)}\left\{\Gamma^{A(r)}-\frac{(-1)^{r}}{m} p_{B} \Gamma^{B A(r)}\right\},
$$

${ }^{c}$ In certain dimensions we could parametrize the manifold $\mathcal{O}_{\mathbf{s}}$ by Weyl or Majorana spinors as well. This, however, leads to different constraints, which depend on each the specific dimension. The parametrization by Dirac spinors does not depend on the dimension explicitly, that seems to be more convenient for the uniform description of the spin in higher dimensions. 


$$
\psi \otimes \widetilde{\psi}=M_{A(r)} \Gamma^{A(r)}
$$

where $\widetilde{\psi}$ is the charge conjugated spinor $\sqrt{a} \widetilde{\psi}^{a}=(\psi C)^{a}$. Hereafter we use the shorthand notation $A(r)=A_{1} \ldots A_{r}$. Tensor $M_{A(r)}=\frac{(-1)^{r(r+1) / 2}}{2^{[d / 2] r} !}\left(\widetilde{\psi} \Gamma_{A(r)} \psi\right)$ obeys equations

$$
\begin{gathered}
M_{[A(r)} Z_{k B]}=0, \quad Z_{k}^{A} M_{A A(r-1)}=0, \quad k=1, \ldots r \\
p^{A} M_{A A(r-1)}=0, \quad M_{A(r)} \sim \lambda^{2} M_{A(r)}
\end{gathered}
$$

Making use of (6) one can express $M_{A(r)}$ in terms of the $Z_{i}$. For these ends it is convenient to introduce another parametrization of $V_{k}$ with the help of $k$-forms $\mathcal{Z}^{k}$ determined from the equations

$$
\mathcal{Z}^{k}\left(\bar{Z}_{1}, \bar{Z}_{2}, \ldots \bar{Z}_{k}\right)=\operatorname{det}\left(Z_{i} \bar{Z}_{j}\right), \quad i, j=1, \ldots k
$$

that establish a relation between $\mathcal{Z}$ and $Z$

$$
\mathcal{Z}_{A(k)}^{k} \sim Z_{1\left[A_{1}\right.} Z_{2 A_{2}} \ldots Z_{\left.k A_{k}\right]}
$$

Tensors $\mathcal{Z}_{A(k)}^{k}$ satisfy the following relations

$$
\begin{gathered}
\mathcal{Z}_{[A(i)}^{i} \mathcal{Z}_{B] B(k-1)}^{k}=0, \eta^{A B} \mathcal{Z}_{A A(i-1)}^{i} \mathcal{Z}_{B B(k-1)}^{k}=0, p^{A} \mathcal{Z}_{A A(k-1)}^{k}=0, \\
\mathcal{Z}_{A(k)}^{k} \sim a_{k} \mathcal{Z}_{A(k)}^{k}, \quad i, k=1, \ldots r, \quad i \geq k, \quad a_{k} \in \mathbf{C} \backslash\{0\}
\end{gathered}
$$

resulting from the definitions (3). Now it is easy to see that the general solution to the equations (6) has the form

$$
M_{A(r)} \sim \mathcal{Z}_{A(r)}^{r}
$$

Thus the $r$-dimensional complex vector subspace $V_{r}$ can be parametrized by the spinor $\psi$ subject to the conditions (4). Making use of (8) we obtain the equivalent parametrization of $\mathcal{O}_{\mathbf{s}}$ in terms of $(r-1)$ complex vectors $Z_{i}^{A}, i=1,2, \ldots r-1, A=0,1, \ldots d-1$ and the spinor $\psi$ subject to equivalence relations

$$
Z_{i}^{A} \sim Z_{j}^{A} \Lambda_{i}^{j}, \quad \psi \sim \lambda \psi, \quad \lambda \in \mathbf{C} \backslash\{0\}
$$

and constraints

$$
\left(p, Z_{i}\right)=0, \quad p_{A} \Gamma^{A} \psi=m \psi \quad,
$$

\footnotetext{
${ }^{d}$ The charge conjugation matrix $C$ is determined from the relation $\Gamma_{A}^{T}=(-1)^{r} C \Gamma_{A} C^{-1}$
} 


$$
\left(Z_{i}, Z_{j}\right)=0, \quad Z_{i A} \Gamma^{A} \psi=0
$$

Here $\Lambda_{i}^{j}$ is a complex non-degenerate upper-triangular $(r-1) \times(r-1)$ matrix. In terms of introduced objects the most general expression for the Kähler potential on $\mathcal{O}_{\mathrm{s}}$ is

$$
\begin{aligned}
& \Phi=\frac{1}{2} \ln \left(\Delta_{1}^{s_{1}} \ldots \Delta_{r-1}^{s_{r-1}} \Delta_{r}^{s_{r}}\right), \\
& \Delta_{i}=Z_{1 A_{1} \ldots} \ldots Z_{i A_{i}} \bar{Z}_{1}^{\left[A_{1}\right.} \ldots \bar{Z}_{i}^{\left.A_{i}\right]}, \quad i=1, \ldots, r-1, \quad \Delta_{r}=(\bar{\psi} \psi)^{2},
\end{aligned}
$$

where $\bar{\psi}^{a}=\left(\psi^{*} \Gamma_{0}\right)^{a}$ is the Dirac conjugated spinor . Note that $\Phi$ depends on $p$ implicitly in view of constraints (12). Under transformations (11) $\Phi$ changes to an additive constant

$$
\delta_{\Lambda, \lambda} \Phi=\sum_{k=1}^{r-1} \ln \left|\Lambda_{1}^{1} \Lambda_{2}^{2} \ldots \Lambda_{k}^{k}\right|^{s_{k}}+\ln |\lambda|^{2 s_{r}}
$$

The direct product structure of $\mathcal{E}$ allows to introduce the one-form $\theta$ being a sum of conventional one-form $p_{A} d x^{A}$ on $\mathcal{E}_{\text {spinless }}$ describing the space-time dynamics of the particle and a one-form on $\mathcal{O}_{\text {s }}$ governing the spinning dynamics. We will put

$$
\theta=p_{A} d x^{A}+* d \Phi,
$$

where the action of the star operator on the complex one-forms is defined as $*\left(\alpha_{I} d z^{I}+\right.$ $\left.\beta_{I} d \bar{z}^{I}\right)=-i\left(\alpha_{I} d z^{I}-\beta_{I} d \bar{z}^{I}\right)$. Notice that $\theta$ is invariant under transformations (11) modulo closed one-form and thus the Hamiltonian action for the system may be chosen as

$$
S=\int_{\gamma} \theta
$$

The extremals of the action (17) coincide with the leaves of ker $d \theta$. By construction ker $d \theta$ is generated by the only vector field $\mathbf{V}=p_{A} \partial / \partial x_{A}$ and hence the tangent vector to the trajectory is proportional to $\mathbf{V}$. This means

$$
\begin{gathered}
\dot{x}^{A}=\mu p^{A}, \quad \dot{p}_{A}=0 \\
\dot{Z}_{i}^{A}=0, \quad \dot{\psi}_{a}=0, \quad \text { (modulo transformations (11)) }
\end{gathered}
$$

where $\mu=\mu(\tau)$ is an arbitrary function of proper time which is fixed after particular choice of a world-line parametrization. Thus the particle moves along the time-like geodesics in the Minkowski space while the internal degrees of freedom do not evolve. 
The interesting feature of the action $(\sqrt{17})$ is that the Dirac equation on $\psi$ subject to the rest constraints $(11,12,13)$ may be covariantly resolved with respect to $p_{A}$. To solve this equation, we multiply it by $\psi_{c}$ and make use of the decomposition (5) together with Fierz identities for spinor bilinears. This results in relations

$$
\begin{array}{r}
p^{A}\left(\widetilde{\psi} \Gamma_{A A(r-1)} \psi\right)=0,\left(\widetilde{\psi} \Gamma_{A A(r)} \psi\right)=\frac{(-1)^{r+1}(r+1)}{m} p_{[A}\left(\widetilde{\psi} \Gamma_{A(r)} \psi\right), \\
p^{A}\left(\widetilde{\psi} \Gamma_{A A(r-1)} \psi\right)=0, \epsilon_{A(r) B C(r)} p^{B}\left(\widetilde{\psi} \Gamma^{C(r)} \psi\right)=(-1)^{1+r(r+1) / 2} r ! m i^{r}\left(\widetilde{\psi} \Gamma_{A(r)} \psi\right)
\end{array}
$$

Contracting the second equation in (19.a) with $\left(\widetilde{\psi} \Gamma^{A(r)} \psi\right)^{*}$ one can express $p_{A}$ via the spinor variables

$$
p_{A}=\bar{p}_{A}\left(\psi, \psi^{*}\right) \equiv(-1)^{r+1} m \frac{\left[\left(\widetilde{\psi} \Gamma_{A B(r)} \psi\right)\left(\widetilde{\psi} \Gamma^{B(r)} \psi\right)^{*}+c . c .\right]}{2\left|\left(\widetilde{\psi} \Gamma_{C(r)} \psi\right)\right|^{2}}
$$

For the odd-dimensional case the similar trick (with $\left(\widetilde{\psi} \Gamma^{B(r)} \psi\right)^{*}$ replaced by $\left.\epsilon^{A(r) C D(r)}\left(\widetilde{\psi} \Gamma_{D(r)} \psi\right)^{*}\right)$ yields

$$
p_{A}=\bar{p}_{A}\left(\psi, \psi^{*}\right) \equiv m \frac{(-1)^{r(r-1) / 2} i^{r} \epsilon_{A B(r) C(r)}\left(\widetilde{\psi} \Gamma^{B(r)} \psi\right)\left(\widetilde{\psi} \Gamma^{C(r)} \psi\right)^{*}}{r !\left|\left(\widetilde{\psi} \Gamma_{D(r)} \psi\right)\right|^{2}}
$$

Since equations (19) form the overdetermined system, the expressions (20) being inserted back into (19) will lead to some consistency conditions which should be imposed on $\psi$ besides the holonomic constraints (13). Note that the mass-shell condition (2) reduces to the purely algebraic relation on $\psi$ which is valid due to these constraints. Thus we obtain the parametrization of the massive hyperboloid in terms of the spinor $\psi_{a}$ subject to the conditions $(11,13)$ and (19) (with $p$ replaced by $\bar{p}$ ). This resembles to the twistor parametrization of the light-cone 17 d which, however, is connected with the division algebras and therefore exists in 3, 4,6 and 10 dimensions only. The derived construction may be considered as its generalization to the case of arbitrary dimensional space-time with the exception that the spinor $\psi$ contains information about both the space-time momentum and intrinsic degrees of freedom.

Substituting $\bar{p}_{A}$ in (16), we immediately derive the Lagrangian of the system

$$
\mathcal{L}=\bar{p}_{A}\left(\psi, \psi^{*}\right) \dot{x}^{A}-i\left(\dot{Z}_{i}^{A} \partial_{A}^{i} \Phi+\dot{\psi}_{a} \partial^{a} \Phi-\text { c.c. }\right)
$$

\footnotetext{
${ }^{d}$ For the models of massless spinning (super)particles which exploit the twistor parametrizations see, e.g. 18
} 
The Lagrangian is obviously invariant under the global Poincaré transformations, reparametrizations of the world-line and changes to a total derivative under the gauge transformations associated with equivalence relations (11)

$$
Z_{i}^{\prime A}(\tau)=Z_{j}^{A}(\tau) \Lambda_{i}^{j}(\tau), \quad \psi_{a}^{\prime}(\tau)=\lambda(\tau) \psi_{a}(\tau)
$$

The global Poincaré symmetry leads to the on-shell conservation of the Hamiltonian counterparts of Poincaré generators $\mathbf{P}_{A}, \mathbf{M}_{A B}$

$$
\begin{gathered}
\mathbf{P}_{A}=\bar{p}_{A}, \quad \mathbf{M}^{A B}=x^{A} \bar{p}^{B}-x^{B} \bar{p}^{A}+S^{A B} \\
S^{A B}=2 i\left\{Z_{i}^{A} \partial^{i B}-\bar{Z}_{i}^{A} \bar{\partial}^{i B}-\left(\Sigma^{A B}\right)_{b}{ }^{a}\left(\psi_{a} \partial^{b}+\bar{\psi}_{a} \bar{\partial}^{b}\right)\right\} \Phi
\end{gathered}
$$

$\left(\Sigma_{A B}\right)_{a}^{b}=-\frac{1}{4}\left[\Gamma_{A}, \Gamma_{B}\right]_{a}^{b}$ being the Lorentz generators in the spinor representation.

Let us now specify the expressions $(19,20)$ to the case of $d=4$. Then the spinning sector is parametrized by one variable $\psi$ defined modulo multiplication by a complex nonzero constant and subject to the Dirac equation. The expression for $p_{A}$ reads

$$
p_{A}=\bar{p}_{A}\left(\psi, \psi^{*}\right) \equiv m \frac{\left(\widetilde{\psi} \Gamma_{A B} \psi\right)\left(\widetilde{\psi} \Gamma^{B} \psi\right)^{*}+c . c .}{2\left|\left(\widetilde{\psi} \Gamma_{C} \psi\right)\right|^{2}}
$$

and the consistency conditions take the form

$$
\bar{p}^{A}\left(\widetilde{\psi} \Gamma_{A} \psi\right)=0, \quad\left(\widetilde{\psi} \Gamma_{A B} \psi\right)=\frac{2}{m} \bar{p}_{[A}\left(\widetilde{\psi} \Gamma_{B]} \psi\right)
$$

In terms of two-component Weyl spinors, $\psi^{t}=\left(\xi_{a}, \bar{\eta}^{\dot{a}}\right)$, the conditions (25) are equivalent to the following one:

$$
\operatorname{Im}\left(\xi_{a} \eta^{a}\right)=0
$$

The Lagrangian (21) is specified as

$$
\mathcal{L}=m \frac{\dot{x}_{A}\left(\sigma_{a \dot{a}}^{A}\right)\left(\xi^{a} \bar{\xi}^{\dot{a}}+\eta^{a} \bar{\eta}^{\dot{a}}\right)}{2\left(\xi_{a} \eta^{a}\right)}+i s \frac{\left(\dot{\xi}_{a} \eta^{a}\right)-\left(\dot{\bar{\xi}}_{a} \bar{\eta}^{a}\right)}{\left(\xi_{a} \eta^{a}\right)}
$$

where $m$ and $s$ stand for the mass and spin of the particle. As is seen, the Lagrangian is invriant under the local projective transformations

$$
\xi \rightarrow \alpha \xi \quad, \quad \eta \rightarrow \bar{\alpha} \eta \quad \forall \alpha \in \mathbf{C} \backslash\{0\}
$$


and becomes singular whenever denominator $\xi_{a} \eta^{a}$ comes to zero. To remove this singularity we can put the partial Lorenz invariant gauge on $\xi$ and $\eta$, breaking the invariance under rescalings with a real $\alpha$ :

$$
\xi_{a} \eta^{a}=m
$$

In this gauge the Lagrangian (27) of $\mathrm{d}=4$ spinning particle takes a quite simple form

$$
\mathcal{L}=\frac{1}{2} \dot{x}_{A}\left(\sigma_{a \dot{a}}^{A}\right)\left(\xi^{a} \bar{\xi}^{\dot{a}}+\eta^{a} \bar{\eta}^{\dot{a}}\right)+\frac{2 s}{m} \operatorname{Im}\left(\xi_{a} \dot{\eta}^{a}\right)
$$

where the spinors $\xi$ and $\eta$ are assumed to be subjected to the one (comlex) holonomic constraint (29). The canonical momenta of the particle resulting from the Lagrangian looks like

$$
p^{A}=\frac{1}{2} \sigma_{a \dot{a}}^{A}\left(\xi^{a} \bar{\xi}^{\dot{a}}+\eta^{a} \bar{\eta}^{\dot{a}}\right)
$$

and automatically satisfies mass-shell condition $p^{2}=m^{2}$ in view of (29). The representation (31) for the momenta of the $d=4$ massive spinning particle in term of two constrained Weyl spinors was originally considered in [19].

Now let us turn back to the original formulation (17) with unresolved momenta and consider the minimal coupling of the particle to an arbitrary background of gravitational and electromagnetic fields. For this end we introduce gauge fields of the vielbein $e_{\mu}^{A}$ and torsion-free spin connection $\omega_{\mu A B}$ associated to the gravity and the electromagnetic potential $A_{\mu}$. Then the minimal covariantization of (17) reads

$$
\begin{gathered}
S=\int \widetilde{\theta} \\
\widetilde{\theta}=\left(p_{A} e_{\mu}^{A}-e A_{\mu}\right) d x^{\mu}+* D \Phi,
\end{gathered}
$$

where $D$ is the Lorentz covariant differential along the particle world-line

$$
\begin{gathered}
D Z_{i}^{A}=d Z_{i}^{A}+d x^{\mu} \omega_{\mu}{ }^{A}{ }_{B} Z_{i}^{B}, \\
D \psi_{a}=d \psi_{a}+d x^{\mu} \omega_{\mu A B} \Sigma_{a}^{A B b} \psi_{b},
\end{gathered}
$$

${ }^{e}$ The higher dimensional generalization of this gauge, removing singularity in expression for the momenta $(20)$, is obvious $-\left|\left(\widetilde{\psi} \Gamma_{C(r)} \psi\right)\right|^{2}=m^{2}$. 
and $e$ is the electric charge. The relations (2, 12) on the momentum $p_{A}$ are still assumed to hold. The action (32) generates the following equations of motion

$$
\begin{gathered}
\dot{x}^{\nu}=\mu e_{A}^{\nu} p^{A}, \quad \frac{D p_{A}}{d \tau}+\mu e F_{A B} p^{B}=(\mu / 4) R_{A B C D} p^{B} S^{C D}, \\
\frac{D Z_{i}^{A}}{d \tau}=0, \quad \frac{D \psi_{a}}{d \tau}=0
\end{gathered}
$$

Here $F_{\mu \nu}$ is the strength tensor of the electromagnetic field and $R_{\alpha \beta \gamma \delta}$ is a curvature of the space-time. Thus the dynamics of the particle in the curved space-time is described by first two equations while the motion in the spinning sector reduces to the parallel transport along the world-line.

To conclude, let us note that the gauge symmetry (22), being required to survive on the quantum level, implies the restriction on the possible values of the parameters $s_{i}$ entering the Kähler potential. Proceeding by analogy with the integer spin case [15] one can show that in quantum theory $s_{i}, i=1, \ldots r-1$ are constrained to be integer and $s_{r}$ (half-)integer numbers.

\section{Quantization}

In this section we will present the covariant quantization of the model described. Let us start with the Lagrangian (21) complemented with the conditions (13) and (19). This Lagrangian leads to the following primary constraints

$$
\begin{gathered}
T_{A}=p_{A}-\bar{p}_{A} \approx 0 \\
\nabla_{A}^{i}=q_{A}^{i}+i \partial_{A}^{i} \Phi \approx 0, \quad \bar{\nabla}_{A}^{i}=\left(\nabla_{A}^{i}\right)^{*} \\
\nabla^{a}=\pi^{a}+i \partial^{a} \Phi \approx 0, \quad \bar{\nabla}^{\dot{a}}=\left(\nabla^{a}\right)^{*}
\end{gathered}
$$

(as well as the above mentioned holonomic ones). Here $p_{A}, q_{A}^{i}$ and $\pi^{a}$ are the momenta conjugated to $x^{A}, Z_{i}^{A}$ and $\psi_{a}$ respectively, $\bar{p}_{A}$ is defined by rels. (20). The system (35) allows a transition to the more suitable basis of constraints where relations (19) have been 
already accounted

$$
\begin{gathered}
T_{i}=\left(p, Z_{i}\right) \approx 0, \quad T_{a}=p_{A} \Gamma^{A} \psi-m \psi \approx 0, \\
\bar{\nabla}_{A}^{i} \approx 0, \quad \bar{\nabla}^{\dot{a}} \approx 0
\end{gathered}
$$

and the complex conjugated constraints are also implied. Stabilization of the constraints yields the mass-shell condition

$$
T=p_{A} p^{A}+m^{2} \approx 0
$$

It should be noted that the constraints (36, 37) are not independent. Moreover, only first class constraints amongst (36) 円 can be covariantly extracted:

$$
\begin{gathered}
\Pi_{j}^{i}=\left(Z_{j}, q^{i}\right)+i \delta_{j}^{i} l_{i}, \quad \bar{\Pi}_{j}^{i}=\left(\Pi_{j}^{i}\right)^{*}, \quad i \geq j, \\
\Pi=\left(\psi_{a} \pi^{a}\right)+i l_{r}, \quad \bar{\Pi}=(\Pi)^{*}, \\
l_{i}=\sum_{k=i}^{r-1} s_{k}, \quad l_{r}=2 s_{r}
\end{gathered}
$$

Constraints $\Pi_{j}^{i}$ and $\Pi$ generate the gauge transformations (11). Following the covariant quantization scheme, the set of variables $(x, p, Z, q, \psi, \pi)$ is associated to the self-adjoint operators acting in a Hilbert space of the particle states. The physical states $|\Psi\rangle$ are singled out from the space of smooth functions on $\mathbf{R}^{d-1,1} \times \mathbf{C}^{(r-1) d} \times \mathbf{C}^{2^{d / 2}}$ by imposing the first class constraint operators and a half of the second class ones. Since the latter cannot be presented explicitly we will impose on the physical states the operatorial counterparts of the expressions (36) (accounting thereby the half of the second class constraints), together with $\hat{\bar{\Pi}}_{j}^{i}$ and $\widehat{\bar{\Pi}}$. As a result, the physical states are annihilated by the operators $\widehat{T}, \widehat{T}_{a}$, $\widehat{T}_{i}, \widehat{\Pi}, \widehat{\Pi}_{j}^{i}, \widehat{\nabla}^{\dot{a}}, \widehat{\nabla}_{A}^{i}$. In the coordinate representation for $(Z, q, \psi, \pi)$ and the momentum one for $(x, p)$

$$
q_{A}^{i} \rightarrow-i \partial_{A}^{i}, \quad \pi^{a} \rightarrow-i \partial^{a}, \quad x^{A} \rightarrow i \partial^{A}
$$

the constraint equations for the wave function $\Psi\left(Z, \bar{Z}, \psi, \psi^{*}, p\right)$ take the following explicit form:

$$
\left(Z_{j}^{A} \partial_{A}^{i}-\delta_{j}^{i} n_{i}\right) \Psi=0, \quad\left(\psi_{a} \partial^{a}-n\right) \Psi=0
$$

\footnotetext{
$f$ Of course, (37) is also first class.
} 


$$
\bar{\partial}_{A}^{i} \Psi+\left(\bar{\partial}_{A}^{i} \Phi\right) \Psi=0, \quad \bar{\partial}^{\dot{a}} \Psi+\left(\bar{\partial}^{\dot{a}} \Phi\right) \Psi=0
$$

The wave function is defined on the surface (12, 13). Note that the constants $n, n_{i}$ may differ from their classical values $l, l_{i}$ due to the different operator ordering prescriptions for $\widehat{\Pi}$ and $\widehat{\Pi}_{j}^{i}$. We fix the ambiguity in the factor ordering by the requirement that the physical wave functions should remain unchanged under the gauge transformations (11) which implies vanishing of $n$ and $n_{i}$. Any other ordering will lead to the unitary equivalent theory. The general solution for (41) reads

$$
\Psi\left(Z, \bar{Z}, \psi, \psi^{*}, p\right)=\exp \left(-\Phi\left(Z, \bar{Z}, \psi, \psi^{*}\right)\right) \Theta(Z, \psi, p)
$$

Substituting (42) in (40) one gets

$$
\left(Z_{j}^{A} \partial_{A}^{i}-\delta_{j}^{i} l_{i}\right) \Theta=0, \quad\left(\psi_{a} \partial^{a}-l_{r}\right) \Theta=0
$$

Since the manifold $\mathcal{O}_{\mathrm{s}}$ is compact the eigenvalues $l_{i}, l_{r}$ prove to be integers with eigenfunctions $\Theta$ represented by the polynomials of the form

$$
\Theta(Z, \psi, p)=\Theta(p)_{A\left(l_{1}\right) B\left(l_{2}\right) \ldots C\left(l_{r-1}\right) a\left(l_{r}\right)} Z_{1}^{A\left(l_{1}\right)} Z_{2}^{B\left(l_{2}\right)} \ldots Z_{r-1}^{C\left(l_{r-1}\right)} \widetilde{\psi}^{a\left(l_{r}\right)}
$$

where we denote $Z_{1}^{A(l)} \equiv Z_{1}^{A_{1}} \cdots Z_{1}^{A_{l}}, \widetilde{\psi}^{a(l)} \equiv \widetilde{\psi}^{a_{1}} \ldots \widetilde{\psi}^{a_{l}} ;$ the symmetry of the indices is described by the Young diagram

\begin{tabular}{|l|l|l|l|}
\hline$A_{1}$ & $A_{2}$ & $\cdots$ & \\
\hline$B_{1}$ & $B_{2}$ & $\cdots$ & $B_{l_{1}}$ \\
\hline \multicolumn{3}{|c|}{} & $\cdots$ \\
\hline$C_{1}$ & $C_{2}$ & $\cdots$ & $C_{l_{r-1}}$ \\
\hline
\end{tabular}

Notice that, by virtue of relations (11, 12, 13), the coefficients $\Theta(p)_{A \ldots C a \ldots}$ are assumed to be $p$-transversal, traceless and $\Gamma$-traceless

$$
p^{A} \Theta(p)_{\ldots A \ldots}=0, \quad \eta^{A B} \Theta(p)_{\ldots A \ldots B \ldots}=0, \quad \Gamma_{a}^{A b} \Theta(p)_{\ldots A \ldots b \ldots}=0
$$

In spinor indices $\Theta$ is subject to the Dirac equation

$$
p_{A} \Gamma_{a}^{A b} \Theta(p)_{\ldots b \ldots}=m \Theta(p) \ldots \ldots
$$

Equations (45, 46) constitute together the full set of $d$-dimensional relativistic wave equations on irreducible massive spin tensor fields which are obtained after Fouriau transform 
of $\Theta(p)$. The space $\mathcal{H}_{m, \mathbf{s}}$ of functions $\Psi$ (42) representing the particle states is endowed with the Hilbert space structure with respect to the following Hermitian inner product

$$
<\Psi_{1} \mid \Psi_{2}>=\int_{p^{2}=-m^{2}} \frac{d \mathbf{p}}{p_{0}} \int_{\mathcal{O}_{\mathbf{s}}} d \mu \bar{\Psi}_{1} \Psi_{2}
$$

where

$$
d \mu=d * d \Phi \wedge d * d \Phi \wedge \ldots \wedge d * d \Phi
$$

is the Liouville measure on $\mathcal{O}_{\mathbf{s}}$. Integration over the spinning degrees of freedom may be performed explicitly resulting with the standard field-theoretical inner product

$$
<\Psi_{1} \mid \Psi_{2}>=N \int_{p^{2}=-m^{2}} \frac{d \mathbf{p}}{p_{0}} \bar{\Theta}(p)_{1}^{A\left(l_{1}\right) B\left(l_{2}\right) \ldots C\left(l_{r-1}\right) a\left(l_{r}\right)} \Theta(p)_{2 A\left(l_{1}\right) B\left(l_{2}\right) \ldots C\left(l_{r-1}\right) a\left(l_{r}\right)}
$$

where $\bar{\Theta}^{\ldots a\left(l_{r}\right)}=\Theta^{* \ldots}{ }_{\dot{a}\left(l_{r}\right)}\left(\Gamma_{0}\right)^{\dot{a}_{1} a_{1}} \ldots\left(\Gamma_{0}\right)^{\dot{a}_{l_{r}} a_{l_{r}}}$ and $N$ is a constant depending on spin of the particle.

Thus the covariant operatorial quantization of the model yields the irreducible representation of the Poincaré group with quantum numbers fixed by the constants originally entering the Kähler potential. We would like also to note that the procedure of geometric quantization provides another way of quantizing these systems with the same result (see [15] where the model of integer spin massive particle is quantized within this approach).

\section{Conclusion}

Let us discuss some possible links between the spinning particle model suggested and some problems of interest in other topics of the field theory and mention some open questions related to the paper results.

In this paper we have extended the description of the integer spin particle [15] to a general (half-)integer case in arbitrary dimension. The key technical step of our construction is the new covariant vector-spinor parametrization for the phase space of $\operatorname{spin} \mathcal{O}_{\mathbf{s}}$, allowing to obtain, upon covariant quantization, arbitrary half-ineger spin representations along with the integer ones. This construction may be thought of as a minimal "spinorization" of the previous one in a sence that only one vector is replaced by a spinor. In principle, it is possible to trade all the vector coordinates for the spinor variables subject to a certain set 
of constraints, which may seem to be more fundamental for dealing with the half-integer spin representations. Then the harmonic expansion of physical wave functions on these variables would define (upon quantization) the irreducible spin-tensor fields similarly to (44). To the best of our knowledge, the respective analysis of irreducibility for the general spin-tensor fields is yet unknown in the higher dimensions, what considerably hinders the determination of an appropriate set of constraints on spinor variables.

As to the problem of interaction between particle and axternal fields, we may mention that, at least at the formal level, it is solved in this paper for the minimal coupling to a general gravity and electromagnetic background fields. The extension can be immediately got to the particle interaction with the dynamical fields by adding the free field Lagrangian in the action. However, the less formal aspect related to the self-accelerating problem [20] remains unclear now in the selfconsistent theory of the particle coupled to the dynamical fields. In ref. [20] has been shown that the selfacceleration of the particle does not occur when it is coupled to a very special spectrum of the fields. This special field spectrum is known for a $d=4$ spinless particle only. For higher dimensions and/or nonzero spin, the solution to the selfacceleration problem could be different. It should be mentioned also that the interaction to the non-abelian gauge fields requires to equip the particle with isospinning degrees of freedom. This could be done, in general, along the same lines as it is performed for a genuine spin in this paper, although the isospin requires different inner manifold instead of $\mathcal{O}_{\mathbf{s}}$. In this way, the isospin-shell conditions should appear as phase space constraints. However, it is unclear from the outset, whether the interplay between spin and isospin in the constraint algebra would be consistent to an arbitrary Yang-Mills background field.

Another problem, where this model may seem to gain some importance, is the relationship between strings and spinning particles. This relationship is commonly known at the level of the quantum string state spectrum which includes an infinite number of massive excitations of various spins. These excitations are usually thought about as states of certain spinning particles. However, it remains yet unclear how the nonzero string modes (subject to the Virasoro constraints) may form the spinning sector of the particle phase space. In other words, the question is how the surface of Virasoro constraints in the string 
phase space is stratified into the spinning particle presymplectic manifolds $\mathcal{E}$. Comprehension of the structure of this stratification seems to be relevant for study of a reduction procedure in string theory. The geometry of the spinning particle phase space, revealed in this paper, forms a basis to study the relationship between strings and particles in this direction.

\section{Acknowledgments}

This work is partially supported by the grant Joint INTAS-RFBR 95-829 and RFBR 9802-16261. A. A. Sharapov appreciates the financial support from the INTAS under the grant YSF 98-153.

\section{References}

[1] A. Frydryszak, Lagrangian models of particles with spin: the first seventy years, hepth/9601020.

[2] P. K. Townsend, Four lectures on M-theory, hep-th/9612121.

[3] M. J. Duff, Supermembranes, CTP-TAMU-61/96, hep-th/9611203.

[4] J. M. Souriau, Structure of dynamical systems (Birkhauser, Boston, 1997).

[5] C. Duval and P. Horvathy, Particles with internal structure: the geometry of classical motions and conservation laws, Ann. of Phys. 142, 10 (1982).

[6] S. M. Kuzenko, S. L. Lyakhovich and A. Yu. Segal, A geometric model of the arbitrary spin massive particle, Int. J. Mod. Phys. A10, 1529 (1995).

[7] S. L. Lyakhovich, A. Yu. Segal, A. A. Sharapov, A universal model of $D=4$ spinning particle, Phys. Rev. D54, 5223 (1996).

[8] S. M. Kuzenko, S. L. Lyakhovich, A. Yu. Segal and A. A. Sharapov, Massive spinning particle on anti-de Sitter space, Int. J. Mod. Phys. A11, 3307 (1996). 
[9] I. V. Gorbunov, V. A. Dolgushev and S. L. Lyakhovich, Galilean spinning particle, to be published in Izv. Vuzov Fiz. in Russian.

[10] S. L. Lyakhovich, A. A. Sharapov, K. M. Shekhter, Spinning particle dynamics on six-dimensional Minkowski space., J. Math. Phys. 38, 4086 (1997).

[11] I. V. Gorbunov, S. M. Kuzenko, S. L. Lyakhovich, On the minimal model of anyons., Int. J. Mod. Phys. A12, 4199 (1997).

[12] S. M. Kuzenko, S. L. Lyakhovich, A. Yu. Segal, Arbitrary superspin massive superparticles., Phys. Lett. B348, 421 (1995).

[13] I.V. Gorbunov, S.L. Lyakhovich, Geometric quantization of $N=2, D=3$ superanyon., Phys. Lett. B423, 293 (1998).

[14] K. Rafanelli, The relativistic spinning : hamiltonian formulation and world line invariance, Phys. Rev. D30, 1707 (1984); M. S. Plyushchay, Relativistic zitterbewegung: the model of spinning particles without Grassmann variables, Phys. Lett. B236, 291 (1990).

[15] S. L. Lyakhovich, A. A. Sharapov and K. M. Shekhter, Massive spinning particle in any dimension I. Integer spins, Nucl. Phys. B537, 640 (1999).

[16] A. Borel, Proc. Nat. Acad. Sci. USA. V.40, 1147, (1954); A. A. Kirillov,Elements of the Theory of Representations (Springer-Verlag, Berlin, 1976).

[17] M. Cederwall, Introduction to division algebras, sphere algebras and twistors, Göteborg-ITP-94-43, hep-th/9310115.

[18] F. Delduc, A. Galperin and E. Sokatchev Lorentz-harmonic (super)fields and (super)particles, Nucl. Phys. B368, 143, (1992); I. Bandos, Sov. J. Nucl. Phys. 51, 906, (1990).

[19] L. C. Biedenharn, H. W. Braden, P. Truini and H. van Dam, Relativistic wavefunctions on spinor spaces, J. Phys A: Math. Gen. 21, 3593 (1988). 
[20] J. W. van Holten, Stability and Mass of Point Particles, Nucl. Phys. B529, 525 (1998). 